\title{
Economics
}

The Open-Access, Open-Assessment E-Journal

Vol. 3, 2009-32 | July 16, 2009 | http://www.economics-ejournal.org/economics/journalarticles/2009-32

\section{Avoiding Extinction: Equal Treatment of the Present and the Future}

\author{
Graciela Chichilnisky \\ Columbia University, New York
}

\begin{abstract}
Equal treatment for the present and the future was required in two axioms introduced in the articles by Chichilnisky of the years 1996 and 1997. These articles provide a characterization of the decision criterion that satisfies the axioms and shows that the two axioms are equivalent to physical limits in the long-run future. The author proves that maximizing discounted utility with a long-run survival constraint is equivalent to maximizing a criterion that treats equally the present and the future. The equal treatment axioms are therefore the essence of sustainable development. The "weight" $\lambda$ given to the long-run future is here identified with the marginal utility of the environmental asset along a path that narrowly misses extinction. An existence theorem is also provided for optimizing according to the welfare criterion that treats equally the present and the future. The author shows that no prior welfare criteria satisfy the axioms for sustainable development introduced in her article of the year 1996.
\end{abstract}

Special issue Discounting the Long-Run Future and Sustainable Development

JEL: H43, Q51, Q54

Keywords: Sustainable development; sustainable preferences; axioms for sustainable development; long-run discounting; dictatorship of the present and the future; extinction, equal treatment for the present and the future; long-run optimization

\section{Correspondence}

Graciela Chichilnisky, Columbia University, New York 10027, USA

e-mail: chichilnisky1@gmail.com

The author is UNESCO Professor of Mathematics and Economics and Director, Program on Information and Resources, Columbia University. Research support from NSF grant No. 9216028 to the Program on Information and Resources at Columbia University, the Stanford Institute for Theoretical Economics (SITE), and from the Institute for International Studies at Stanford University is gratefully acknowledged, as are the comments of Y. Baryshnikov, P. Ehrlich, P. Eisenberger, C. Figuieres, S. C. Kolm, D. Kennedy, D. Kreps, L. Lauwers, C. Perrings, D. Starrett, L. van Liedekierke, P. Milgrom, J . Roberts, R. Wilson, and H. M. Wu. Special thanks are due to Kenneth Arrow, Peter Hammond, Geoffrey Heal, Mark Machina, Robert Solow, Richard Howarth, Charles Figuieres, Mabel Tidball and an anonymous referee. The basic research for this article was prepared for an invited presentation at a seminar on Reconsideration of Values at the Stanford Institute for Theoretical Economics, organized by K. J. Arrow in July 1993. It was also an invited presentation at the Intergovernmental Panel on Climate Change (IPCC) Seminar in Montreux, Switzerland, March 1994, as a lead author of the IPCC, at a Seminar on Inconmensurability of Values at Chateaux du Baffy, Normandy, April 1994, and at the Graduate School of Business of Stanford University in May 1994, in 2007 at the School of Economics, Oslo University in Norway, Department of Mathematical Statistics Columbia University, 2007, and GREQAM Universite de Marseille France and LAMETA at the University of Montpellier, France, December 2008. 


\section{Introduction}

Global warming and the plight of extinguishing species are attracting increasing public attention, and leading to calls for new forms of economic development. But sustainable development is hardly a new issue. The need for development that satisfies the basic needs of present and future generations was introduced 30 years ago $^{1}$ (Chichilnisky 1977a, 1977b; Herrera et al. 1976), it was developed by the ILO and the World Bank in country studies, and reaffirmed by international vote as a global development priority 17 years ago $^{2}$ (Brundtland 1987) ${ }^{3}$. Yet the challenge to achieve sustainable development remains today as elusive as ever. A change in preferences is essential. What this article shows is that once we become aware of new long term physical constraint on resources, such as the possible extinction of a species, this invokes new behavioral axioms introduced in Chichilnisky (1996) requiring equal treatment of the present and the future - and we behave according to the decision criterion they imply. It is essential therefore that we focus on the limits we face as a species. The article shows that the "equal treatment" axioms are equivalent to preferences that reflect awareness of physical limits. Therefore the new axioms are the essence of sustainable development.

Implementing sustainable development is a moving target that requires more than public attention and a change in values and preferences. It requires a solid analysis of sustainability with the level of clarity and substance of neoclassical theory, to support the practical scope and the current widespread use of markets and cost benefit analysis. ${ }^{4}$ The crux of the matter is how, through markets, we can define economic values that go beyond immediate individual gain and encompass the needs of future generations. ${ }^{5}$ This motivation led the author ${ }^{6}$ to propose in 1993 two

\footnotetext{
${ }^{1}$ The concept of development based on the satisfaction of basic needs was introduced by the author in the mid 1970s in the Bariloche Model and several other publications (Chichilnisky 1977a, 1977b; Herrera et al. 1976) and became the cornerstone of efforts to define sustainable development (see Chichilnisky et al. 1998) in the 1987 Brundland Report (Brundtland 1987), which uses the concept explicity, defining sustainable development as 'development that satisfies the needs of the present and the future'.

${ }^{2}$ At the Earth Summit of Rio de Janaeiro 1992, where the concept of Basic Needs was voted by 150 nations as the main concept of sustainable development.

${ }^{3}$ Brundland's Report in 1987 (Brundtland 1987) defined 'sustainable development' on the basis of basic needs, as "a form of development that satisfies the needs of the present without preventing the future from satisfying its own needs." This was voted by 150 nations as a global priority in the Earth Summit of 1992 in Rio de Janeiro. Yet Basic Needs and Sustainable Development are still to achieve mainstream status.

${ }^{4}$ Solow (1992) pointed out that discussion of sustainability has been mainly an occasion for the expression of emotions and attitudes, with very little formal analysis of sustainability or of sustainable paths for a modern industrial economy. One purpose of Chichilnisky (1996, 1997, 1999, 2000, 2002, 2009a, 2009c) was to resolve this problem.

${ }^{5}$ Standard cost-benefit analysis discounts and undervalues the future (Chichilnisky 1996, 1997; Hammond 1993). It is therefore biased against policies designed to provide benefits in the very long run. An example is the evaluation of projects for the safe disposal of waste from a nuclear power plant. Another is policies designed for the prevention of global warming. The benefits of both may be years into the future. The costs, however, are here today. In these cases, the inherent asymmetry between the treatment of present and future makes it hard to justify investment decisions that large numbers of individuals and organizations clearly feel are well merited.

${ }^{6}$ Neoclassical theory of choice and preference theory was developed in the first part of the 20th
} 
axioms to define sustainable development requiring equal treatment for the present and the future, and to derive the decision criteria that they imply (Chichilnisky 1996, 1997). The two axioms require that neither the present nor the future should be "dictatorial". This article takes the matter further by solving outstanding issues that were not covered in the original treatment: (i) what is the practical basis for the new axioms, where do they come from? Exactly why and how do individuals change their preferences and start taking into consideration the long term future? (ii) how to identify in practice the weight given to the long-run future, and (iii) how to ensure the existence of sustainable solutions. The three issues were raised in the subsequence literature on the topic (Chichilnisky 1994, 1995, 1998a, 1998b; Beltratti et al. 1995; Heal 2000; Lauwers 1993, 1997) and are resolved here. In a nutshell we show in Theorem 3 that the new axioms are in fact a rational response to a new objective reality, a reaction to new long term physical constraints that we face today and did not exist before, and that the weight given to the long-run future represents the potential losses from the extinction of the resource in the long run. I also show that the existence of solutions is guaranteed by the inability of physical systems to adapt beyond a certain speed of response.

The implications of these results is clear. It is indisputable the world economy has changed, facing us with physical limits that did not exist before. We have become aware of the possibility of the extinction of our species. This article shows that this awareness leads us to behave according to the new equal treatment axioms (Chichilnisky 1996) and the decision criterion that they imply.

\section{Changing Preferences}

It is worth underscoring the enormous physical transition that took place since the middle of the 20th century, a period in which most economic analysis of preferences was developed. For the first time in history, humans dominate the planet and consume resources in a way that can alter the planet's climate, its water bodies and its biological mix. Fossil fuel energy used for production since the second world war emitted carbon that could alter irreversibly the earth's climate with catastrophic consequences. Biologists see the loss of biodiversity during the last sixty years as one of the four or five largest incidents of destruction of life on the planet, 1,000 times larger than fossil records show. ${ }^{7}$ Of the 5,487 known species of mammals - our relatives $-25 \%$ have become extinct ${ }^{8}$ sending a somber message to the rest.

A voracious use of resources since World War II originated largely in the industrial countries, and was accompanied by increasing discrepancies in resource consumption and welfare between industrial and developing countries, the North and the South (Chichilnisky 1998c). The problem has been high in the international

century and is based in axioms from which 'present discounted value' and 'expected utility anlysis' were derived. They were created by Tjallings Koopmans for the case of choice over time and by Von Neumann for the case of choice under uncertainty and grew to achieve the status of common knowledge (Arrow 1953; Hammond 1993; Chichilnisky 1997, 1996; Heal 2000).

${ }^{7}$ According to the UN 2000 Millenium Report (United Nations 2000), see also Chichilnisky (2009b).

${ }^{8}$ See Schipper (2008).

www.economics-ejournal.org 
agenda since the 1992 United Nations Earth Summit in Rio de Janeiro, where the issue of sustainable development ${ }^{9}$ emerged as one of the most urgent topics of international policy and one hundred and fifty nations endorsed UN Agenda 21 requiring new patterns of sustainable development that can satisfy the basic needs of the present and the future. ${ }^{10}$ Yet little progress has been achieved since then. The use of biodiversity and fossil fuels has increased rapidly and water is now the most scarce resource in the world. ${ }^{11}$

The "equal treatment" axioms for the present and the future have not been debated, partly because there is general agreement now that economics has to take account of the long run (Cline 1992; Cropper et al. 1994; Dutta 1991; Hammond 1993; Heal 2000; Krautkramer 1985; Lauwers 1993; Lowenstein and Elster 1992, Lowenstein and Thaler 1989; Solow 1992). Yet three outstanding issues emerged in the ensuing literature ${ }^{12}$ and are resolved here. The first is how to explain the practical meaning of the new axioms - where do they come from? Theorem 3 shows that if we are aware of long term constraints, then we must accept the two "equal treatment" axioms proposed in 1993 (Chichilnisky 1996) and the decision criteria that they imply. The new axioms are equivalent to the awareness of physical limits in the long-run future.

A second related issue is how to compute the "weight" $\lambda$ that is assigned to the long-run future by a sustainable preference. ${ }^{13}$ The initial representation theorem provided in Chichilnisky (1996) had a degree of freedom for this parameter, which remained undefined. This can be considered a useful feature of the theory, and parallels the two degrees of freedom that appear in traditional discounted utility analysis, namely the 'instantaneous utility' and the 'discount factor' that are used to define discounted utility, and were left undefined. Yet it can be useful to identify the value of the "weight" $\lambda$ in specific cases, to show how it can be computed in practice. In this article we consider the case of renewable resources that can become extinct when overused, a timely issue since $25 \%$ of all known mammals are now extinct (Schipper 2008). Theorem 3 establishes that the optimizing according to

\footnotetext{
${ }^{9}$ At the 1992 United Nations Earth Summit in Rio de Janeiro, sustainable development emerged as one of the most urgent subjects for international policy. As already pointed out, one hundred and fifty participating nations endorsed UN Agenda 21, proposing as part of its policy agenda sustainable development based on the satisfaction of basic needs in developing countries.

${ }^{10}$ As pointed out above, the development criterion based on the satisfaction of basic needs, was introduced and developed empirically by the author in 1976 in the 'Bariloche Model' and in several scientific publications in the mid 1970s (Chichilnisky 1977a, 1977b; Herrera et al. 1976), and given further impetus in 1987 when the Brundtland Commission proposed that "sustainable development is development that satisfies the needs of the present without undermining the needs of the future" Brundtland (1987, chap. 2, para. 1).

${ }^{11}$ The global use of resources and the difference of consumption between poor and rich nations has become more acute since 1992, Chichilnisky (2009b).

${ }^{12}$ For example, at a recent presentation during a Seminar in Cargese, France in the Spring of 2007 Charles Perrings argued that my criterion of sustainable preferences (which arises from my two axioms) was less applicable because we did not know the exact value of the parameter $\lambda$ that appears in front of the long run future utility. A similar point was made by a Columbia University colleague, Peter Eisenberger. Both seem misplaced concerns, as explained above, since the flexibility in the parameter is a valuable aspect of the theory.

${ }^{13}$ This is represented by a real number " $\lambda$ " that appears in the representation of a sustainable utility, see Chichilnisky $(1996,1997)$.
} 
the new axioms and the attendant preferences, is equivalent to optimizing based on a new physical constraint on the resource in the long term, and that the factor $\lambda$ is the marginal utility of the resource at the point of extinction This is the point where the resource is presumably most valuable. Theorem 4 resolves the issue of existence of optimal solutions, using Sobolev's Theorem that provides conditions of "bounded variation" on the consumption paths as extended in Chichilnisky $(1997,1977 \mathrm{c})$. The last section examines a list of previously used preferences and shows that none satisfy the axioms of sustainability defined here, so this theory of sustainable development represents genuine change. The results provided here add a new angle to the initial axioms and the sustainable preferences they imply. These results help to compute solutions and explain further the emergence of sustainable preferences as awareness about the depth and scope of biodiversity destruction and the risk of potentially catastrophic climate change in the long-run future. In other words, to change our preferences what is needed is to increase awareness of the new long term constraints on resources.

\section{Experimental Evidence on How We Value the Long Run}

In the last twenty years many experiments have measured how people value the long run (see, e.g., Lowenstein and Thaler 1989; Cropper et al. 1994; and the references in Lowenstein and Elster 1992). Their findings clash with the traditional discounted utility approach. In practice, people value the present and the future differently from the theoretical predictions of standard analysis; the present and the future are treated more evenhandedly. Typically we discount the future, but our trade-off between today and tomorrow blurs as we move into the future. Tomorrow acquires increasing more importance as time progresses. It is as if we viewed the future through a curved lens. The relative weight given to two subsequent periods in the future is inversely related to their distance from today. The period-to-period rate of discount is inversely related to the distance into the future. The experimental evidence shows that rate of discount between period $t$ and period $t+1$ decreases with $t$. Interestingly, studies of human responses to sound summarized in the WeberFechner law (Chichilnisky 1996; Heal 2000), indicate similar responses to changes in sound intensity: The human ear responds to sound stimuli in an inverse relation to the initial stimulus. Resent research by Lieberman and Trope (2008) confirms the psychology of transcending the here and now and shows that our mental space traverses temporal, spatial, and social distance through the same abstract processing of information. Recent empirical research shows considerable similarity in the way people mentally transverse these different types of distances, and how we evaluate, predict and plan near and distant situations.

How to explain this experimental evidence, our sensitivity to time, and how to integrate it into a criterion of optimality? Several interesting alternatives to the discounted utility analysis have been proposed. So far none had reached the clarity and consistency of the discounted utilitarian criterion used in cost-benefit analysis, nor its analytical tractability. Prominent examples are the "overtaking 
criterion", (Weizacker 1967), Ramsey's criterion (Ramsey 1928) and "basic needs" (Chichilnisky 1977a, 1977b). However, these criteria are incomplete, failing to rank many reasonable paths. The ordering induced by the overtaking criterion cannot be represented by a real valued function, making it impractical to use. As a result, they lack the corresponding "shadow" prices to evaluate costs and benefits in an impartial fashion. These criteria therefore fail on practical grounds. The last section of this article examines these and other criteria that were previously used.

In 1993, Chichilnisky proposed simple axioms that capture the concept of sustainability, and derived the welfare criterion which they imply, see Chichilnisky $(1996,1997)$. The criterion that emerges is complete and analytically tractable. In optimization it leads to shadow prices which can be used for a "sustainable costbenefit analysis". The new axioms provide internal consistency and ethical clarity. They imply a more symmetric treatment of generations in the sense that neither the "present" nor the "future" should be favored over the other. They neither accept the romantic view which relishes the future without regards for the present, nor the consumerist view which ranks the present above all. The axioms lead to a complete characterization of sustainable preferences, which are sensitive to the welfare of all generations and offer an equal opportunity to the present and to the future. Trade-offs between present and future consumption are allowed. The existence and characterization of 'sustainable preferences' appeared first in Chichilnisky $(1996)^{14}$ and sustainable preferences were shown to be a natural extension of the "equal treatment criterion" for finitely many generations, in the sense that the optimal solutions for such preferences approach the "turnpike" of an 'equal-weight finite horizon optimization problem as the horizon increases (Chichilnisky 1997). Theorem 3 of Chichilnisky (1997) showed that sustainable preferences match the experimental evidence in these cases, in the sense that they imply a rate of discount that decreases and approaches zero as time goes to infinity, and Theorem 4 investigated the relationship between the optimal paths according to sustainable preferences and discounted utilitarianism in an extension of the classical Hotelling problem of the optimal depletion of an exhaustible resource. Theorem 5 in (Chichilnisky 1997) showed that sustainable optima can be quite different from discounted optima, no matter how small is the discount factor.

\section{Two Axioms for Sustainable Development}

The two axioms of Chichilnisky (1996) are non-dictatorship properties (Arrow 1953; Chichilnisky 1982). Axiom 1 requires that the present should not dictate the outcome in disregard for the future: it requires sensitivity to the welfare of generations in the distant future. Axiom 2 requires that the future should not dictate the outcome in disregard for the present, and thus requires sensitivity to the present. To offer a formal perspective a few definitions are required. Each generation is represented by an integer $g, g=1,2, \ldots$ An infinitely lived world obviates the need to make decisions contingent on an unknown terminal date. Generations could overlap or

\footnotetext{
${ }^{14}$ An alternative name for "sustainable preferences" was suggested by Robert Solow in personal communication: "intertemporally equitable preferences".
}

www.economics-ejournal.org 
not. Indeed agents could be infinitely long-lived and evaluate development paths for their own futures. For ease of comparison, I adopt a formulation which is as close as possible to that of Chichilnisky (1996) and to the standard neoclassical model. Each generation $g$ has a preference that can be represented by a utility function $u_{g}$ for consumption of $n$ goods, some of which could be environmental goods such as water, or soil, so that consumption vectors are in $R^{n}$, and $u_{g}: R^{n} \rightarrow R$. The availability of goods in the economy can be constrained in a number of ways, for example by a differential equation which represents the growth of the stock of a renewable resource and/or the accumulation and depreciation of capital. We ignore for the moment population growth, although this issue can be incorporated with little change in the results, at the cost of more notation. ${ }^{15}$

The space of all feasible consumption paths is indicated $F=\left\{x: x=\left\{x_{g}\right\}, g=\right.$ $\left.1,2, \ldots, x_{g} \in R^{n}\right\}$.We chose a utility representation so that each generation's utility

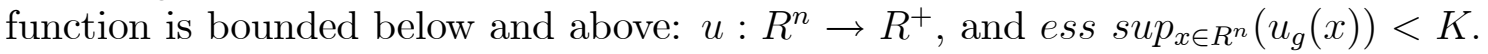
This choice is not restrictive: it was shown by Arrow (1971) that when ranking infinite streams of utilities as done here one should work with bounded utility representations since doing otherwise could lead to paradoxes. ${ }^{16}$ In order to eliminate some of the most obvious problems of comparability I normalize the supremum of utilities to be 1 , Arrow (1953) and Chichilnisky (1982). ${ }^{17}$ In this case we are concerned with fairness across generations, see also Solow (1992), Lauwers (1993, 1997), Chichilnisky (1977a, 1982), Beltratti et al. (1995), Chichilnisky (1994, 1995). The space of feasible utility streams is therefore $Q=\left\{\alpha: \alpha=\left(\alpha_{g}\right), g=1,2, \ldots, \alpha_{g}=\right.$ $\left.u_{g}\left(x_{g}\right) \in R, g=1,2, ..\right\}$.

\section{The Present and the Future}

Each utility stream is a bounded sequence of positive real numbers. The space of all utility streams is contained as a bounded set in in the space of all infinite bounded sequences of real numbers, denoted $F=l_{\infty} \cdot{ }^{18}$ A welfare criterion $W$ should rank elements of $F$, for all possible $f \in F$. The welfare criterion $W$ is complete if it is represented by an increasing real valued function defined on all bounded utility streams $W: l_{\infty} \rightarrow R$. It is called sensitive or increasing, if whenever a utility stream $\alpha$ is obtained from another $\beta$ by increasing the welfare of some generation, then $W$ ranks $\alpha$ strictly higher than $\beta$.

\footnotetext{
${ }^{15}$ Population growth and utilitarian analysis are well known to make an explosive mix, which is however outside the scope of this paper.

${ }^{16}$ The need to work with bounded utility representation is pointed out by K. Arrow (1971), who requires boundedness to solve the problem that gave rise to Daniel Bernouilli's famous paper on the "St. Petersburg paradox".

${ }^{17}$ A preference admits more than one utility representation; among these one chooses a bounded representation. Utility functions $u$ that are nonnegative and all stare a common bound, which I assume without loss of generality to be 1 .

${ }^{18}$ Formally $: l \infty=\left\{y: y=\left\{y_{g}\right), g=1, \ldots: y_{g} \in R^{+}\right.$and $\left.\sup _{g}\left\|y_{g}\right\|<K\right\}$. Here $\|$. $\|$ denotes the absolute value that is used to endow $l \infty$ with a standard Banach space structure, defined by the norm $\|$.$\| . The space of sequences l \infty$ was first used in economics by G. Debreu (1954).
} 


\subsection{The Present}

Intuitively, the present is represented by all the utility streams which have no future: for any given utility stream $\alpha$, its "present" is represented by all finite utility steams which are obtained by cutting a off after any number of generations. Formally:

DEFINITION. For any utility stream $\alpha$ and any integer $K$ let $\alpha_{K}$ be the " $K$-cut off" of the sequence $\alpha$, the sequence whose coordinates up to and including the $K$-th are equal to those of $\alpha$, and zero after the $K$-th.

DEFINITION. The present consists of all feasible utility streams which have no future i.e., it consists of the cutoffs of all utility streams, as defined above in Definition 1.

\subsection{The Future}

By analogy, for any given utility stream a, its "future" is represented by all infinite utility streams which are obtained as the "tail" resulting from cutting a off for any finite number of generations.

DEFINITION. The $K$-th tail of $\beta$ is the sequence whose coordinates up to and including the $K$-th are zero and equal to those of $\beta$ after the $K$-th generation.

\subsection{No Dictatorship of the Present}

DEFINITION. A welfare function $W: l_{\infty} \rightarrow R$ gives a dictatorial role to the present, ${ }^{19}$ and is called a dictatorship of the present, if $W$ is insensitive to the utility levels of all but a finite number of generations, i.e., if $W$ is only sensitive to the "cutoffs" of utility streams, and it disregards the utility levels of all generations from some generation on. Formally, for every $\alpha, \beta, W(\alpha)>W(\beta)$ if and only if there exists an $N>0, N=N(\alpha, \beta)$ such that $W\left(\alpha^{\prime}\right)>W\left(\beta^{\prime}\right)$ for any $\alpha^{\prime}, \beta^{\prime}$ that differ from $\alpha$ and $\beta$ only in their elements after $N$, namely $\forall g<N, \alpha_{g}=\alpha_{g}^{\prime}$ and $\beta_{g}=\beta_{g}^{\prime}$.The following axiom eliminates dictatorships of the present:

Axiom 1: No dictatorship of the present.

\subsection{No Dictatorship of the Future}

DEFINITION. A welfare function $W$ gives a dictatorial role to the future, and is called a dictatorship of the future, if $W$ is insensitive to the utility levels of any finite number of generations, or equivalently it is only sensitive to the utility levels of the "tails" of utility streams. Formally, for every $\alpha, \beta, W(\alpha)>W(\beta)$ if and only if there exists an $N>0, N=N(\alpha, \beta)$ such that $W\left(\alpha^{\prime}\right)>W\left(\beta^{\prime}\right)$ for any $\alpha^{\prime}, \beta^{\prime}$ that differ from $\alpha$ and $\beta$ only in their elements prior to $N$, namely $\forall g>N, \alpha_{g}=\alpha_{g}^{\prime}$ and $\beta_{g}=\beta_{g}^{\prime}$. The welfare criterion $W$ is therefore only sensitive to the utilities of "tails" of streams, and in this sense the future always dictates the outcome independently of the present. The following axiom eliminates dictatorships of the future:

Axiom 2: No dictatorship of the future.

\footnotetext{
${ }^{19}$ The representability of the order $W$ by a real valued function can be obtained from more primitive assumptions, such as, for example, transitivity, completeness, and continuity conditions.
} 


\subsection{Sustainable Preferences}

DEFINITION. A sustainable preference is a complete sensitive continuous preference $W: l_{\infty} \rightarrow R$ satisfying Axioms 1 and 2: it is therefore neither a dictatorship of the present nor a dictatorship of the future.

\section{Existence and Characterization of Sustainable Preferences}

Why is it difficult to rank infinite utility streams? Ideally one would give equal weight to every generation. For example, with a finite number $N$ of generations, each generation can be assigned weight $1 / N$. But when trying to extend this criterion to infinitely many generations one encounters the problem that, in the limit, every generation is given zero weight since $\lim 1 / N=0$. What is done usually to solve this problem is to attach more weight to the utility of near generations, and less weight to future ones. An example is of course the sum of discounted utilities. Discounted utilities give a bounded welfare level to every utility stream which assigns each generation the same utility. Two numbers can always be compared, so that the criterion so defined is clearly complete. However, the sum of discounted utilities is not even-handed: it disregards the long-run future. It was shown in Chichilnisky $(1996,1997)$ that it is a dictatorship of the present as defined above. Another solution is the criterion defined by the long-run average of a utility stream, a criterion used frequently in repeated games. However, this criterion is not even-handed either: it is biased in favor of the future and against the present. It is insensitive to the welfare of any finite number of generations. ${ }^{20}$ Here matters stood for some time. Asking for the two axioms together, the non dictatorship of the present and the non dictatorship of the future, as required by the Axioms above, appears almost as if it would lead to an impossibility theorem. Not quite.

Let us reason again by analogy with the case of finite generations. The number of generations one can assign weights which decline into the future, and then assign some extra weight to the last generation. This procedure, when extended naturally to infinitely many generations, is neither dictatorial for the present nora dictatorship of the future. It is similar to adding to a sum of discounted utilities, the long-run average of the whole utility stream. Neither part of the sum is acceptable on its own, but together they are. This is Theorem 1 in Chichilnisky (1996). Theorem 2 in Chichilnisky (1996) proved that a similar procedure gives a complete characterization of all continuous sustainable preferences. The first part of Theorem 1 establishes that the sum of a dictatorship of the present plus a dictatorship of the future is in fact neither. The first part is sensitive to the present, and the second is sensitive to the future. Furthermore such a sum admits trade-offs between the welfare of the present and of the future. Theorem 1 in Chichilnisky (1996) made this reasoning rigorous, see also Theorem 1 below and the Appendix. The second part of Theorem 1 below shows that all known criteria of optimality used until now

\footnotetext{
${ }^{20}$ Other interesting incomplete intergenerational criteria which have otherwise points in common with sustainable preferences are found in Asheim (1988, 1991).
} 
fail to satisfy the axioms postulated here, and is proved in the Appendix. Therefore the sustainable preferences defined here perform a role satisfied by no previously used criterion. What is perhaps more surprising is that the sustainable welfare criteria identified here, namely the sum of a dictatorship of the present and one of the future, exhaust all the continuous utilities that satisfy my two axioms. This means that any continuous sustainable preference must be of the form just indicated. This is Theorem 2 below, see also Chichilnisky (1996). The results of Theorem 3 below add another aspect to the characterization of sustainable preferences - it shows that a sum of a dictatorship of the present and one of the future represents the essence of sustainable development, since maximizing such preferences is equivalent to the awareness of a new resource constraint in the long run into the standard discounted utility framework used until now.

\subsection{Existence of Sustainable Preferences}

Theorem 1: There exists a sustainable preference $W: l_{\infty} \rightarrow R$, i.e., a preference which is sensitive and does not assign a dictatorial role to either the present or the future

$$
W(\alpha)=(1-\lambda) \sum_{g=1}^{\infty} \mu_{g} \alpha_{g}+\lambda \Phi(\alpha)
$$

where $1>\lambda>0, \forall g, \mu_{g}>0, \sum_{g=1}^{\infty} \mu_{g} \alpha_{g}<\infty$, and $\Phi(\alpha)$ is the function $\Phi(\alpha)=$ $\lim _{g \rightarrow \infty} \alpha_{g}$ when this limit exists extended to all of $l_{\infty}$ by means of Hahn Banach's theorem. ${ }^{21}$ None of the welfare criteria used until now in economics satisfies these new axioms: (a) the sum of discounted utilities, for any fixed discount factor no matter how small, (b) Ramsey's criterion, (c) the overtaking criterion, (d) lim inf, (e) long-run averages, ${ }^{22}$,(f) Rawlsian rules, (g) basic needs and (h) The Green Golden Rule $g^{*}$ (Beltratti et al. 1995).

\section{Proof:}

For a proof see the Appendix and also Chichilnisky (1996, 1997). The Appendix includes a definition of the prior criteria and shows how they fail the sustainability or equal treatment axioms.

${ }^{21}$ The linear map " $\lim _{g} \alpha_{g}$ " is defined by using the Hahn-Banach theorem, as follows: define first the function on the closed subset of $l_{\infty}$ denoted $\tilde{l_{\infty}}$ consisting of those sequences $\alpha_{g}$ that have a limit. On $\tilde{l_{\infty}}$ the function is defined as the sequence's $\operatorname{limit}_{\lim } \alpha_{g}$; the function is then extended continuously to all sequences in the whole space $l_{\infty}$ by using the Hahn-Banach theorem, which ensures that such an extension exists, it is continuous and can be constructed while preserving the norm of the initial function defined on the closed subspace of convergent subsequences, see Chichilnisky (1996) and Yoshida (1974).

${ }^{22}$ For example the two sequences $(1,0,0,111,0,0,0,0,1,1,1,1,1,0,0,0,0,0,0,1 \ldots \ldots)$ and $(0,1,1,0,0,0,1,1,1,1,0,0,0,0,0,1,1,1,1,1,1,0 . . .+)$ are not comparable according to the long-run averages criterion. 


\subsection{Characterization of Sustainable Preferences}

The existence theorem presented above has been extended to provide a complete characterization of sustainable preferences in Chichilnisky (1996):

Theorem 2: Let $W: l_{\infty} \rightarrow R$ be a continuous sustainable preference. Then $W$ is of the form

$$
W(\alpha)=(1-\lambda) \sum_{g=1}^{\infty} \mu_{g} \alpha_{g}+\lambda \Phi(\alpha)
$$

where $1>\lambda>0, \forall g, \mu_{g}>0, \sum_{g=1}^{\infty} \mu_{g} \alpha_{g}<\infty$ and where $\Phi(\alpha)$ is a finitely additive measure on $l_{\infty}{ }^{23}$

\section{Proof:}

For a proof see the Appendix and also Chichilnisky $(1996,1997)$.

\section{How We Change Preferences: Origin of the New Axioms}

This section explains how preferences change and the origin of the axioms defined in Chichilnisky (1996). The theorems below prove that under certain conditions maximizing a sustainable preference is mathematically equivalent to maximizing a standard preference with a new additional constraint at infinity, a constraint that did not exist before. ${ }^{24}$ The implication of this is that as we become aware of new physical constraints in the long run and consider these in making decisions, this leads us to behave according to the two axioms Chichilnisky (1996) that treat equally the present and the future, and to optimize the decision criteria that they imply.

The Theorem presented below resolves an outstanding issue that was pointed out about sustainable preferences: it identifies in practical terms a parameter, the "weight" $\lambda$ that sustainable preferences assign to the long term future, appearing in the characterization Theorem 2 above and in Chichilnisky (1996). The parameter $\lambda$ can be identified with the marginal value of an environmental asset, computed at the point of its possible extinction.

Consider a 'standard' optimization problem: maximizing a continuous linear function $W(\alpha)=\int_{R} \alpha(x) f(x) d x=\int_{R} \alpha(x) d \nu$ where $f(x) \in L_{1}^{+}$so $\nu$ is a finite measure on $R$. In the following such a $W$ is called a standard discounted utility preference over infinite streams. Theorem 3 establishes under certain conditions the equivalence between two problems: (1) optimizing a sustainable preference $W^{*}$ and (2) optimizing a standard preference $W$ with an added constraint in the long run, or 'at infinity'. For simplicity, we separate the existence problem from the characterization of solutions. Theorem 3 characterizes the solutions, assuming that

\footnotetext{
${ }^{23}$ For definitions and examples of purely finitely additive measures, see Chichilnisky (1996) and the Appendix. A purely finitely additive measure can be defined on the line $R$ as well as on a finite interval such as $[0,1]$.

${ }^{24}$ For simplicity in the following we consider a simple preferences that are the sum of discounted utilities, and where $\Omega=l_{\infty}$. More general results are possible at the cost of more notation.
} 
they exist. Theorem 4 establishes conditions that are sufficient for the existence of solutions.

Theorem 3 suggests how sustainable preferences emerge from standard preferences: upon the consideration of new constraints at infinity, for example, from new survival constraints in the long run on a renewable resource such as an animal species, a constraint that was not considered or did not exist before. When the constraint represents a requirement to avoid extinction of the species, then the parameter $\lambda$ that appears in the sustainable preference representing the weight give to the long-run future, is identified with the marginal utility of this renewable resource at the point of its possible extinction.

We need a few definitions. In this section we use both discrete time $t=1,2, \ldots$ and continuous time $t \in R$ to facilitate comparison with the literature, using a single notation for simplicity: let $L_{\infty}(R)$ to be the space of essentially bounded real valued functions on $R$, and $\widetilde{L_{\infty}}(R)$ the closed subspace of functions that have a limit at infinity, $\lim _{g \rightarrow \infty} \alpha_{g}<\infty$ a.e; with discrete time (replacing $R$ by the integers $Z$ ) these two spaces $L_{\infty}(Z)$ and $\tilde{L_{\infty}}(Z)$ become the spaces of bounded sequences of real numbers $l_{\infty}$ used in previous sections, and $\tilde{l_{\infty}}$ the subspace of sequences that have a limit at infinity, respectively. Observe that the space $L_{\infty}(R) \subset L_{2}(R, \nu)=$ the Hilbert space of all square integrable measurable real valued functions with the measure $v$ on $R$ when the measure $\nu$ is finite on $R$ as above, see Chichilnisky (1977c). Observe also that the space $L_{2}(R, \nu)$ contains functions that are neither bounded, nor go to zero at infinity, since the measure $v$ is finite by assumption. We now define 'weighted' Sobolev spaces, which were introduced in optimal growth and general equilibrium theory in Chichilnisky $(1976,1977$ c, 1986) and in Chichilnisky and Zhou (1998). $C^{k}(R)$ is the space of $k$ - times differentiable functions with the $C^{k}$ norm $\|f\|_{k}=S u p_{x \in R}\left|f(x), D f(x), \ldots D^{k} f(x)\right|, 0 \leqq k \leqq \infty$, and for any integer $s \geq 0$ define the Sobolev norm $\|$. $\|^{s}$ as $\|f\|^{s}=\int \sum_{0 \leqq k \leqq s}\left|D^{k} f(x)\right|^{2} d x$. The Sobolev Space $H^{s}(R)$ is the completion of $C^{\infty}(R)$ under this norm. This space includes measurable functions that may be discontinuous. The 'weighted' Sobolev space $H^{s}(R, v)$ is the Sobolev space $H^{s}(R)$ when $R$ is endowed with a finite measure $v$ as above. All Sobolev spaces are Hilbert spaces. Weighted Sobolev spaces contain unbounded real valued function on $R$. Using Sobolev's Theorem as extended to weighted Sobolev spaces in Chichilnisky (1977c), one can ensure the continuity and smoothness of solutions, and also that closed and bounded subsets of $H^{s}$ are compact as needed for the existence of solutions. In most function spaces, such as $L_{\infty}$, a closed bounded set is not generally compact. ${ }^{25}$

Sobolev Theorem: (Sobolev 1963; Chichilnisky (1976, 1986); Chichilnisky and Kalman 1980). Let $s>k+1 / 2$. Then $H^{s}(R) \subset C^{k}(R)$ and the inclusion is continuous and compact.

Under the above conditions:

Theorem 3: Assume that for some $K>0$ the two problems below have unique solutions. Then the two optimization problems are equivalent:

\footnotetext{
${ }^{25}$ In fact, the closed unit ball is never compact in indifinite dimensional Banach spaces.
} 
Problem 1: Optimize a standard concave continuous increasing preference $W$ : $L_{\infty}(R) \rightarrow R$

$$
\operatorname{Max}_{\alpha} W(\alpha) \quad \text { where } W(\alpha)=\int_{R} \alpha(x) \cdot d v=\int \alpha(x) f(x) d x, f(x) \in L_{2}(R, v)
$$

with the following additional constraint at infinity for $K>0$

$$
\infty>\lim _{g \rightarrow \infty} \alpha(x) \geq K
$$

and

Problem 2: Optimize a corresponding sustainable preference ${ }^{26} W^{*}(\alpha): \widetilde{L_{\infty}} \rightarrow R$ with no additional constraint at infinity, namely: ${ }^{27}$

$$
\operatorname{Max} \underset{\alpha \subset \tilde{L_{\infty}}}{\sim} W^{*}(\alpha)=\operatorname{Max} \underset{\alpha \in \tilde{L_{\infty}}}{\sim}(1-\lambda) W(\alpha)+\lambda \Phi(\alpha)
$$

or equivalently

$$
\operatorname{Max} \underset{\alpha \subset \tilde{L_{\infty}}}{\sim} W^{*}(\alpha)=\operatorname{Max} \underset{\alpha \in L_{\infty}}{\sim} W(\alpha)+\mu \Phi(\alpha)
$$

where

$$
\lambda=\frac{\mu}{1-\mu} .
$$

The parameter $\mu$ can be identified as the 'marginal utility at the point of extinction' of the resource, namely the Frechet derivative:

$$
\mu=\left.\frac{\partial}{\partial \alpha} W^{*}(\alpha)\right|_{\alpha^{*}}
$$

computed at a path $\alpha^{*}$ that satisfies $\infty>\lim _{g \rightarrow \infty} \alpha(x)=K$, where $K>0$ is as in Problem 1. ${ }^{28}$

\section{Proof:}

The space $L_{\infty}(R)$ is a Banach space that is contained in the weighted Hilbert space $L_{2}(R, \nu)=H^{0}(R, v)$ because by assumption $v$ is a finite measure on $R$ see Chichilnisky (1977c). The preference $W: L_{\infty} \rightarrow R$ is a continuous linear function that is extendable to all $L_{2}(R, v)$, an extension denoted also $W: L_{2}(R, v) \rightarrow R$, since by the the assumptions on Problem 1, the 'kernel' in $W$ is in $L_{2}$, namely $f(x) \in L_{2}(R, v)$.

Since $W$ is linear and continuus, $W$ is Frechet differentiable on $L_{2}(R, v)$, and we may use calculus in Hilbert spaces to define the Frechet derivative of $W$ which

\footnotetext{
${ }^{26}$ As defined in Theorem 2 above.

${ }^{27}$ The function $\Phi(\alpha)$ was defined in Theorem 2 as an extension of the function $\lim _{x \rightarrow \infty} \alpha(x)$ to all of $L_{\infty}$.

${ }^{28}$ In Problem 2, $\frac{\partial}{\partial \alpha} W(\alpha)$ represents the Frechet derivative of the function $W$ with respect to the variable $\alpha \in l_{\infty}$, and $\Phi(\alpha)$ is a purely finitely additive measure on $l_{\infty}, 0<\lambda<1$.
} 
is the analogue of standard derivatives in euclidean space. Under these conditions to establish the result one may apply Theorem 1 p. 243 of Luenberger (1969) and Theorem 1 of Chichilnisky and Kalman (1979, p. 495 ) that characterizes optimal solutions to contrained optimization problems in weighted Hilbert spaces.

To provide an intuitive motivation it is helpful to supply a simple finite dimensional analogue, since $R^{N}$ is also a Hilbert space. Observe that Problem 1 above is formally equivalent in finite dimensional cases to optimizing a concave continuous increasing function defined on $R^{N}$ (for some $N>0$ ) together with a constraint on the values of the last period, $N$, namely Problem 1 in N-dimensional euclidean space can be written as:

$$
\operatorname{Max}_{\alpha \in R^{N}} W(\alpha)=\sum_{g=1}^{N} \partial_{g} \alpha_{g}
$$

with a constraint

$$
\alpha(N)=\alpha_{N} \geq K>0 .
$$

Observe that for any real number $\mu>0$, an optimum of a function $F(\alpha)$ is also an optimum of the function $\mu . F(\alpha)$ and viceversa.

By standard results of constrained maximization in euclidean spaces, since $W$ is increasing, the maximum of the above problem is achieved at a boundary of the constraint set, so there exists a $\mu>0$ for which the maximum of Problem 1 coincides with the maximum of the following problem:

$$
\operatorname{Max}_{\alpha \in R^{N}} W(\alpha)+\mu\left(\alpha-\alpha_{N}\right)
$$

By the above observation, for any $\lambda, 0<\mu<1$, this is a a maximum of

$$
(1 / 1-\mu)\left(W(\alpha)+\mu\left(\alpha-\alpha_{N}\right)\right.
$$

namely

$$
\left.\operatorname{Max}_{\alpha \in R^{N}}(1-\lambda) W(\alpha)+\lambda\left(\alpha-\alpha_{N}\right)\right]
$$

where

$$
\lambda=\frac{\mu}{1-\mu} \text { and }(1-\lambda)=\frac{1-2 \mu}{1-\mu} .
$$

$0<\lambda<1$, where the expression $\operatorname{Max}_{\alpha \in R^{N}}(1-\lambda) W(\alpha)+\lambda\left(\alpha-\alpha_{N}\right)$ has the form of a "sustainable preference" as in Problem 2. Resolving this (finite dimensional) Problem 1 implies

$$
\frac{\partial}{\partial \alpha}(W)=\mu\left(\alpha_{N}-K\right)
$$


and

$$
\mu=\left.\frac{\partial}{\partial \alpha} W(\alpha)\right|_{\alpha_{N}=K}
$$

where, as above,

$$
\lambda=\frac{\mu}{1-\mu} .
$$

The argument above exhibits the formal equivalence between a solution of Problems 1 and 2 above in finite dimensional euclidean N-space. A similar result holds in Hilbert spaces, which are the closest analog of euclidean space in infinite dimensions. Under the conditions of Theorem 3 we may apply Theorem 1 of Luenberger (1969, p. 243), and Theorem 1 of Chichilnisky and Kalman (1979, p. 495) on contrained optimization in infinite dimensional vector spaces, obtaining a similar result as that presented above for the infinite dimensional case. Consider Problem 1 defined on the weighted Hilbert space $L_{2}(R, v)$. If for some $K>0, \alpha^{*}$ resolves the (contrained) problem

$$
\operatorname{Max}_{\alpha} W(\alpha)
$$

with

$$
\lim _{x \rightarrow \infty} \alpha(x) \geqslant K
$$

then there exists a $\mu$ such that $\alpha^{*}$ is also optimal for the problem

$$
\operatorname{Max}_{\alpha \in L_{2}} W(\alpha)+\mu\left(\alpha-\lim _{x \rightarrow \infty} \alpha(x)\right)
$$

or, equivalently, as shown above $\alpha^{*}$ is optimal for the problem

$$
\operatorname{Max}_{\alpha \in L_{2}}(1-\lambda) W(\alpha)+\lambda\left(\alpha-\lim _{x \rightarrow \infty} \alpha(x)\right)
$$

where

$$
\lambda=\frac{\mu}{1-\mu}
$$

$0<\lambda<1$, which is Problem 2 above.

Observe that the function $\Phi(x)=\lim _{x \rightarrow \infty} \alpha(x)$ that appears in the second term of $W^{*}$ in Problem 2 is well defined and (by definition) a linear function, under the assumptions on the contraint space $\widetilde{L_{\infty}}(R)$. The parameter $\mu$ is given by the Frechet derivative

$$
\mu=\left.\frac{\partial}{\partial \alpha} W(\alpha)\right|_{\lim _{g \rightarrow \infty} \alpha_{g}=K} .
$$

Therefore under the conditions, resolving Problem 1 for an appropriate $K>0$ is 
equivalent to resolving Problem 2 for an appropriate $\lambda=\frac{\mu}{1-\mu}$ that depends on $K$. This completes the proof.

Observe that in Theorem 3 we have assumed the existence of unique solutions to both Problems. The following result establishes sufficient conditions for existence. Problem 1 in Theorem 3 is standard in the literature and conditions for the existence of solutions have been established with or without concavity; for the non concave case see Chichilnisky (1977c). What remains to be established is the existence of solutions to Problem 2, which is not standard since the criterion to be optimized is a sustainable preferences satisfying the axioms of equal treatment of the present and the future. We see below that existence with sustainable preferences is assured when there are bounds to the variation of the feasible paths, such as limits in the change of a species population through time. These seem natural conditions for existence, since biological populations have adjustment limitations, and therefore limited variability, through time. For this purpose, Sobolev's theorem is particularly useful as it provides compactness conditions that are similar to "bounded variation" conditions.

As before, let $W^{*}: L_{\infty} \rightarrow R$ be a continuous sustainable preference satisfying the two Axioms presented above.

Theorem 4: If the feasible set of paths $\Omega \subset L_{\infty}$ is bounded and closed in the norm of $H^{s}$ and $s \geq 1$, then there exists a solution to the sustainable optimization Problem 2 within $\Omega$.

\section{Proof:}

The proof establishes existence of solutions by a continuity and compactness argument. It requires to establish compactness of the feasible set of functions $\Omega$ in the same topology where the function $W^{*}$ is continuous. The result is obtained by using bounded variation conditions on the functions in $\Omega$, more specifically Sobolev's theorem as stated above. First observe that the function $W^{*}$ is continuous when restricted to the space $C^{o}(R)$, where $C^{o}(R) \subset L_{\infty}(R)$ is the subspace of $L_{\infty}(R)$ of continuous bounded real valued functions on $R$. The next step is to observe that $L_{\infty}(R) \subset H^{0}(R)=L_{2}(R)$ since the measure $v$ is finite, cf. Chichilnisky (1977c). By the assumptions and Sobolev's theorem the feasible subset $\Omega \subset L_{\infty}(R)$ consists of continuous real valued functions on $R$ since by Sobolev's theorem $H^{s} \subset C^{o}$ when $s>1 / 2$ and we assumed that $s \geq 1$. Recall that the inclusion $H^{1} \subset C^{0}$ is compact by Sobolev's theorem and the set $\Omega$ is closed and bounded by assumption. Therefore the set $\Omega$ is compact in $C^{0} \subset L_{\infty}$. Finally, by assumption $W^{*}$ is continuous on $L_{\infty}$. Therefore under the conditions there exist a solution to Problem 2 of Theorem 3 in the set $\Omega$.

\section{The Axiom of Choice}

It is worth mentioning that the correspondence between Problem 1 and Problem 2 established in Theorem 3 above is itself equivalent to the well known Axiom of Choice at the foundations of Mathematics. The reason is as follows. The Frechet derivative in Theorem 3 exists because the function $W$ is by construction continuous and linear (Chichilnisky 1996). However, the actual construction of a function that represents the Frechet derivative is not possible in all cases, since this requires the use 
of a Hahn Banach theorem, and in general Hahn Banach theorem depends on using the Axiom of Choice for the construction of a separating hyperplane. Kurt Godel (1963) established that the most general solutions to the construction problems just mentioned (e.g. the hyperplanes in Hahn Banach Theorem) are independent of the rest of the axioms of mathematics: they can neither be established nor negated, as are the Continuum Hypothesis and the Axiom of Choice.

\section{No Prior Criteria are Sustainable Preferences}

Although the axioms presented here are reasonable, all preferences considered in the literature so far fail to satisfy them.

\section{Proposition 1}

The following criteria for evaluating time paths fail the sustainability axioms (Chichilnisky 1997) including completeness and sensitivity:

(a) the sum of discounted utilities, for any fixed discount factor no matter how small, because it is always a dictatorship of the present, as established in Chichilnisky (1996),

(b) Ramsey's criterion, which is seriously incomplete and therefore does not satisfy the definition of a sustainable preference see Chichilnisky $(1996,1997)$,

(c) the overtaking criterion, because it is also incomplete, see Chichilnisky (1997),

(d) lim inf, which is a dictatorship of the future Chichilnisky (1997),

(e) long-run averages, because it is a dictatorship of the future and also incomplete, ${ }^{29}$

(f) Rawlsian rules because it is insensitive Chichilnisky (1997) as it ranks equally two paths that treat equally those who are worst off, no matter how everyone else (including infinitely many generations) is treated,

(g) basic needs satisfies continuity and the two equal treatment axioms but it is insensitive as defined in Chichilnisky (1997), since it ranks equally two paths that have the same infimum - even though one may assign a much higher utility to many (even to infinitely many) generations.

(h) The Green Golden Rule $g^{*}$ defined in Beltratti et al. (1995) as a stationary path $g^{*}=\left\{c^{*}, s^{*}\right\}$ that achieves the maximum utility level that is sustainable forever, that is, $g^{*}=\max u(c, s)$ subject to $c<R(s)$.

For definitions and proofs see the Appendix.

\footnotetext{
${ }^{29}$ For example the two sequences $(1,0,0,111,0,0,0,0,1,1,1,1,1,0,0,0,0,0,0,1 \ldots \ldots)$ and $(0,1,1,0,0,0,1,1,1,1,0,0,0,0,0,1,1,1,1,1,1,0 . . . \quad)$ are not comparable according to the long-run averages criterion.
} 


\section{APPENDIX}

\subsection{Continuity}

In practical terms the continuity of $W$ is the requirement that there should exist a sufficient statistic for inferring the welfare criterion from actual data. This is an expression of the condition that it should be possible to approximate as closely as desired the welfare criterion $W$ by sampling over large enough finite samples of utility streams. Continuity of a sustainable criterion function $W$ is not needed in Theorem 1; it is used solely for the characterization in Theorem 2. Continuity is defined here in terms of the standard topology of 'sup norm' defined by $\|f\|$ $=\sup _{x \in R}|f(x)|$. This topology was first used in economics by Debreu (1954).

DEFINITION. A sustainable preference is a complete continuos preference defined on $L_{\infty}$ satisfying Axioms 1 and 2 .

\subsection{Previous Welfare Criteria}

To facilitate comparison, this subsection defines some of the more widely used prior welfare criteria. A function $W: l_{\infty} \rightarrow R$ is called a discounted sum of utilities if it is of the form: $W(\alpha)=\sum_{g=1}^{\infty} \mu_{g}, \alpha_{g}$ where $\forall g, \alpha_{g} \geq 0$ and $\sum \mu_{g}<\infty ; \mu_{g}$ is called a discount factor.

Ramsey's welfare criterion (Ramsey 1928) ranks a utility stream $\alpha=\left(\alpha_{g}\right) g=$ $1,2, \ldots$ above another $\beta=\left(\beta_{g}\right) g=1,2, \ldots$ if the utility stream $\alpha$ is "closer" to the bliss path, namely to the sequence $t=(1, l, \ldots, 1 \ldots)$, than is the sequence $\beta$. Formally: $\sum_{g=1}^{\infty} 1-\alpha_{g}<\sum_{g=1}^{\infty} 1-\beta_{g}$.

A Rawlsian rule (Rawls 1971) ranks two utility streams according to which has a higher infimum value of utility for all generations. This is a natural extension of the criterion proposed initially by Rawls (1971). Formally: a utility stream a is preferred to another if $\inf \left(\alpha_{g}\right)>\inf \left(\alpha_{g}\right)$. The criterion of satisfaction of basic needs introduced in Chichilnisky $(1977 \mathrm{a}, 1977 \mathrm{~b})$ ranks a utility stream $\alpha$ over another $\beta$ if the time required to meet basic needs is shorter in $\alpha$ than in $\beta$. Formally: $T(\alpha) \leqq T(\beta)$ where $T(\alpha)=\min \left\{t: \alpha_{g}>b\right\}$, for a given $b$ which represents basic needs. The overtaking criterion (von Weizacker 1967)) ranks a utility stream $\alpha$ over another $\beta$ if $\alpha$ eventually leads to a permanently higher level of aggregate utility than does $\beta$. Formally: $\alpha$ is preferred to $\beta$ if $\exists n$ :

$$
\forall M>N, \sum_{g-1}^{M}\left(\alpha_{g}\right)>\sum_{g-1}^{M}\left(\beta_{g}\right)
$$

The long-run average criterion can be defined in our context as follows: a utility stream $\alpha$ is preferred to another $\beta$ if in average terms, the long-run aggregate utility achieved by $\alpha$ is larger than achieved by $\beta$. Formally: $\exists N, K>0: \frac{1}{T}\left(\sum_{g-M}^{T+M}\right.$ $\alpha_{g}>\frac{1}{T}\left(\sum_{g-M}^{T+M} \beta_{g}\right) \forall T>N$ and $M>K$. 


\subsection{Countable and Finitely Additive Measures}

DEFINITION. Let $\left(S, \sum\right)$ denote the field of all subsets of a set $S$ with the operations of unions and intersections of sets. A real valued, bounded additive set function on $\left(S, \sum\right)$ is one which assigns a real value to each element of $\left(S, \sum\right)$, and assigns the sum of the values to the union of two disjoint sets.

DEFINITION. A real valued bounded additive set function is called countably additive if it assigns the countable sum of the values to a countable union of disjoint sets.

\section{EXAMPLE 1.}

Probability measures on the real numbers, $R$, or on the integers $Z$, are typical examples of countably additive functions. Any sequence of positive real numbers $\left\{\lambda_{g}\right\} g=1 .,, .$. , such that $\sum \lambda_{g}<\infty$ defines a countably additive measure $\mu$ on the integers $Z$, by the rule $\mu(A)=\sum_{g \in A} \lambda_{g}, \forall A \subset Z$.

DEFINITION. A real valued bounded additive set function $\varphi$ on $\left(S, \sum\right)$ is called purely finitely additive (see Yosida 1974; and Yosida and Hewitt 1952) if whenever a countably additive function $\nu$ satisfies: $\forall A \in\left(S, \sum\right), \nu(A)<\varphi(A)$ then $\nu(A)=0$ $\forall A \in\left(S, \sum\right)$. This means that the only countably additive measure which is absolutely continuous with respect to a purely finitely additive measure is the measure that is identically zero.

\section{EXAMPLE 2.}

Any real valued linear function $V: l_{\infty} \rightarrow R$ defines a bounded additive function $V$ on the field $\left(Z, \sum\right)$ of subsets of the integers $Z$ as follows: $\forall A \subset Z, \hat{V}(A)=$ $V\left(\alpha^{A}\right)$ where $\alpha^{A}$ is the "characteristic function" of the set $A$, namely the sequence defined by $\alpha^{A}=\left\{\alpha_{g}^{A}\right\}, g=1,2, \ldots$ such that $\alpha_{g}^{A}=1=1$ if $g \in A$ and $\alpha_{g}^{A}=0$ otherwise.

\section{EXAMPLE 3.}

Typical purely finitely additive set functions on the field of all subsets of the integers, $\left(Z, \sum\right)$, are the liminf function on $l_{\infty}$, defined for each by $\lim \inf (\alpha)=\lim$ inf $\left\{\alpha_{g}\right\} g=1,2, \ldots$ Recall that the lim inf of a sequence is the infimum of the set of points of accumulation of the sequence. The "long-run averages" function is another example: it is defined for each a $\alpha \in l_{\infty}$ by

$$
\lim _{K, N \rightarrow \infty}\left(\frac{1}{K} \sum_{g=N}^{K+N} \alpha_{g}\right) .
$$

It is worth noting that a purely finitely additive set function $\phi$ on the field of subsets of the integers $\left(Z, \sum\right)$ cannot be represented by a sequence of real numbers in the sense that there exists no sequence of positive real numbers, $\lambda=\left\{\lambda_{g}\right\}$ which defines $\phi$, that is, there is for no $\lambda$ such that $\forall A \subset Z, \phi(A)=\sum_{n \in A} \lambda_{n}$. For example the function $\lim$ inf $: l_{\infty} \rightarrow R$, defines a purely finitely additive set on a function on the field of subsets of the integers which is not representable by a sequence of real numbers. 


\subsection{Proof of Theorem 1}

\section{Proof:}

See also Chichilnisky (1996). To establish the existence of a sustainable preference it suffices to exhibit a function $W: l_{\infty} \rightarrow R$ satisfying the two axioms. For any $\alpha \in l_{\infty}$ consider $W(\alpha)=\sum \lambda_{g} \alpha_{g}+\lim \left(\alpha_{g}\right) g=1,2 . . . \forall \alpha=\left(\alpha_{g}\right)$, with a well defined $\lim _{g \rightarrow \infty} \alpha_{g}$, and $\lim \left(\alpha_{g}\right)$ extended to all of $l_{\infty}$ using Hahn Banach's theorem. $W$ satisfies the axioms because it is a well-defined, non-negative, increasing function on $l_{\infty}$; it is not a dictatorship of the present (Axiom 1) because its second term makes it sensitive to changes in the "tails" of sequences; it is not a dictatorship of the future (Axiom 2) and because its first term makes it sensitive to changes in "cutoffs" of sequences. The next task is to show that the following welfare criteria do not define sustainable preferences: (a) Ramsey's criterion, (b) the overtaking criterion, (c) the sum of discounted utilities, (d) lim inf, (e) long-run averages (f) Rawlsian criteria, and (g) basic needs. The Ramsey's criterion defined above fails because it is not a well-defined real valued function on all of $l_{\infty}$ and cannot therefore define a complete order on $l_{\infty}$. To see this it suffices to consider any sequence for which the sum does not converge. For example, let $\alpha=\left(\alpha_{g}, g=1,2, \ldots\right.$ where $\left.\forall g, \alpha_{g}=(g-1) / g\right)$. Then $\alpha_{g} \rightarrow 1$ so that the sequence approaches the "bliss" consumption path $\beta=(1,1, \ldots, 1 \ldots)$. The ranking of $\alpha$ is obtained by the sum of the distance between a and the bliss path. Since $\lim _{N \rightarrow \infty} \sum_{g=1}^{N}\left(1-\alpha_{g}\right)$ does not converge, Ramsey's welfare criterion does not define a sustainable preference as defined. The overtaking criterion defined above is not a well-defined function of $l_{\infty}$, since it cannot rank those pairs of utility streams in which neither $\alpha$ overtakes $\beta$ nor $\beta$ overtakes $\alpha$. Figure 2 in Chichilnisky (1997) exhibits a typical pair of utility streams which the overtaking criterion fails to rank. The long-run averages criterion defined above and the lim inf criterion defined above fail on the grounds that neither satisfies Axiom 2; both are dictatorships of the future. Finally any discounted utility criterion of the form $\mathrm{W}(\alpha)=\sum \alpha_{g} \lambda_{g}$ for $\forall g, \lambda_{g}>0$ and $\sum \lambda_{g}<\infty$ is a dictatorship of the present, as shown in Chichilnisky (1996) and therefore fails to satisfy Axiom 1. Finally the Rawlsian welfare criterion and the criterion of satisfaction of basic needs do not define sustainable preferences: the Rawlsian criterion defined above fails because it is not sensitive to the welfare of many generations: only to that of the less favored generation. Basic needs has a similar drawback.

\subsection{Proof of Theorem 2}

\section{Proof:}

Consider a continuous independent sustainable preference that satisfies Axioms 1 and 2, so there exists a utility representation for $W$. The welfare criterion $W: l_{\infty} \rightarrow R$, defines a non-negative, continuous linear functional on $l_{\infty}$. As seen above in Example 3, such a function defines a non-negative, bounded, additive set function denoted $W$ on the field of subsets of the integers $Z,\left(Z, \sum\right)$. The representation theorem of Yosida and Hewitt (Yosida 1974; Yosida and Hewitt 1952) establishes that every non-negative, bounded, additive set function on $\left(S, \sum\right)$, the field of subsets $\sum$ of a set $S$ can be decomposed into the sum of a non-negative countably additive measure $\mu$ and a purely finitely additive, which is a non-negative 
set function $\phi$ on $\left(S, \sum\right)$. It follows from this theorem that $W$ can be represented as the sum of a countably additive measure $\mu$ and a purely finitely additive measure $\phi$ on the integers $Z$. It is immediate to verify that this is the representation provided above. To complete the characterization proof it suffices to show that neither $\mu$ nor $\phi$ are identically zero. This follows from Axioms 1 and 2: we saw above that discounted utility is a dictatorship of the present, so that if $\phi \approx 0$, then $W$ would be a dictatorship of the present, contradicting Axiom 1. If on the other hand $\mu \approx$ 0 , then $W$ would be a dictatorship of the future because all purely finitely additive measures are, by definition, dictatorships of the future, contradicting Axiom 2. Therefore neither $\phi$ nor $\mu$ can be identically zero. This completes the proof of the theorem. 


\section{References}

Arrow, K. J. (1953). Social Choice and Individual Values. Cowles Foundation Monographs. New York: Wiley 1964.

Arrow, K.J. (1971). Aspects of the Theory of Risk-Bearing. Yrjo Jahnsson Lectures, Yrjo Jahonsson in Saatio, Helsinki.

Asheim, G. (1988). "Rawlsian Intergenerational Justice as a Markov-Perfect Equilibrium in a Resource Economy". Review of Economic Studies 55 (3): 469-484.

Asheim, G. (1991). "Unjust Intergenerational Allocations". Journal of Economic Theory 54 (2): 350-371.

Beltratti, A., G., Chichilnisky, and G. M. Heal (1995). "The Green Golden Rule". Economics Letters 49 (2): 175-179.

Brundtland, G. H. (1987). Report of the World Commission on Environment and Development: Our Common Future. Oxford: Oxford University Press.

Chichilnisky, G. (1976). "Manifolds of Preferences and Equilibria". PhD Dissertation, UC Berkeley, www.chichilnisky.com.

Chichilnisky, G. (1977a). "Economic Development and Efficiency Criteria in the Satisfaction of Basic Needs". Applied Mathematical Modeling 1 (6): 290-297.

Chichilnisky, G. (1977b). "Development Patterns and the International Order". Journal of International Affairs 31 (2): 275-304.

Chichilnisky, G. (1977c). "Nonlinear Functional Analysis and Optimal Economic Growth". Journal of Mathematical Analysis and Applications 61 (2): 504-520.

Chichilnisky, G. (1982). "Social Aggregation Rules and Continuity" . Quarterly Journal of Economics 97 (2): 337-352.

Chichilnisky, G. (1986). "Topological Complexity of Manifolds of Preferences" Chapter 8, Contributions to Mathematical Economics in Honor of Gerard Debreu (eds. Werner Hildenbrand and Andreu Mas-Colell). North Holland, Amsterdam, New York.

Chichilnisky, G. (1994). "The Environment and the Long Run: A Comparison of Different Criteria" (with A. Beltratti and G. M. Heal). Richerche Economiche 48: 319-340.

Chichilnisky, G. (1995). "Sustainable Growth and the Green Golden Rule" (with A. Beltratti and G. M. Heal). Presented at conference on Sustainable Growth, Paris, May 1993, published in I. Goldin and A. Winters (eds), The Economics of Sustainable Development, OECD, Paris. 
Chichilnisky, G. (1996). "An Axiomatic Approach to Sustainable Development". Social Choice and Welfare 13 (2): 231-257.

Chichilnisky, G. (1997). "What is Sustainable Development". Land Economics 73 (4): 467-491.

Chichilnisky, G. (1998a). "Sustainable Use of Renewable Resources" (with A. Beltratti and G.M. Heal), Chapter 2.1, Sustainablity: Dynamics and Uncertainty, (eds. G. Chichilnisky, G.M. Heal, and A.Vercelli). Kluwer Academic Publishers, The Netherlands.

Chichilnisky, G. (1998b). "Uncertain Future Preferences and Conservation" (with A. Beltratti and G.M. Heal), Chapter 3.4 in Sustainabilitv: Dynamics and Uncertainty (eds. G. Chichilnisky, G. M. Heal, and A. Vercelli). Kluwer Academic Publishers, The Netherlands.

Chichilnisky, G. (1998c). "Sustainable Development and North-South Trade" in Protection of Global Biodiversity: Converging Strategies, (eds. Lakshman D. Guruswamy and Jeffrey A. McNeely), Duke University Press, Durham and London, p. 101-117.

Chichilnisky, G. (1999). "Intergenerational Choice: A Paradox and A Solution" in Topology and Markets (G. Chichilnisky ed.) American Mathematical Society and Fields Institute for Mathematical Sciences, Vol 22: 99-110 (with Y. Baryshnikov).

Chichilnisky, G. (2000). "An Axiomatic Approach to Choice Under Uncertainty with Catastrophic Risks". Resource 65 Energy Economics 22 (3): 221-231.

Chichilnisky, G. (2002). "Catastrophic Risk" in Encyclopedia of Environmetrics, edited by Abdel H. El-Shaarawi and Walter W. Piegorsch, published by John Wiley \& Sons, Ltd, Chichester, UK, Volume 1, pp. 274-279.

Chichilnisky, G. (2006). "Catastrophic Risks: The Need for New Tools, Financial Instruments and Institutions", Symposium on the Privatization of Risk-Social Science Research Council, New York June.

Chichilnisky, G. (2009a). "The Limits of Econometrics: Nonparametric Estimation in Hilbert Spaces". Econometric Theory 25 (4): 1070-1086.

Chichilnisky, G. (2009b). Beyond the Global Divide: From Basic Needs to the Knowledge Revolution, to appear 2009-10.

Chichilnisky, G. (2009c). "The Topology of Fear". Journal of Mathematical Economics, Special Issue on the 2007 NBER General Equilibrium Conference in Honor of Gerard Debreu. Forthcoming.

Chichilnisky, G., and P. Kalman (1979). "Comparative Statics and Dynamics of Optimal Choice Models in Hilbert Spaces". Journal of Mathematical Analysis and Applications 70 (2): 490-504. 
Chichilnisky, G., and P. J. Kalman (1980). "Application of Functional Analysis to Models of Efficient Allocation of Economic Resources". Journal of Optimization Theory and Applications 30 (1): 19-32.

Chichilnisky, G., and Y. Zhou (1998). "Smooth Infinite Economies". Journal of Mathematical Economics 29 (1): 27-42.

Chichilnisky, G. and K. Sheeran (2009). Saving Kyoto, New Holland, UK.

Chichilnisky, G., G. Heal and S. Vercelli (1998). Sustainability, Dynamics and Uncertainty. Kluwer Academic Publishers.

Cline, W. R. (1992). The Economics of Global Warming. Washington, DC: Institute for International Economics.

Cropper, M. L., S. K. Aydede, and P. Portney (1994). "Preferences for Life Saving Programs: How the Public Discounts Time and Age". Journal of Risk and Uncertainty 8: 243-265.

Dasgupta, P., and G. Heal (1979). Economic Theory and Exhaustible Resources. Cambridge: Cambridge University Press.

Debreu, G. (1954). "Valuation Equilibrium and Pareto Optimum". Proceedings of the National Academy of Sciences 40 (July): 588-592.

Dutta, P. (1991). "What Do Discounted Optima Converge to?" Journal of Economic Theory 55 (1): 64-94.

Godel, K. (1963). The Independence of the Continuum Hypothesis. Mathematics Monographs, Princeton University Press. American Mathematical Society, Providence R.I.

Hammond, P. J. (1993). "Is There Anything New in the Concept of Sustainable Development?" Presented at the conference The Environment after Rio, Courmayeur, Italy, 10-12 February.

Heal, G. (2000). Valuing the Future: Economic Theory and Sustainability. Columbia University Press.

Herrera, A., H. Scolnik, G. Chichilnisky et al. (1976). Catastrophe or New Society: A Latin American World Model (The Bariloche Model). Ottawa, Canada: International Development Research Center.

Krautkramer, J. (1985). "Optimal Growth, Resource Amenities and the Preservation of Natural Environments". Review of Economic Studies 52 (1): 153-170.

Lauwers, L. (1993). "Infinite Chichilnisky Rules". Economics Letters 42 (4): 349352.

Lauwers, L. (1997). "A Note on Weak-Chichilnisky Rules". Social Choice and Welfare 14 (2): 357-359. 
Lieberman, N., and Y. Trope (2008). "The Psychology of Transcending the Here and Now". Science 21 (November) 322: 1201-1205.

Loewenstein, G., and J. Elster, eds. (1992). Choice Over Time. New York: Russell Sage Foundation.

Loewenstein, G., and R. Thaler (1989). "Intertemporal Choice". Journal of Economic Perspectives 3 (4): 181-193.

Luenberger, D.G. (1969). Optimization by Vector Spaces Methods. Wiley, New York.

Ramsey, F. (1928). "A Mathematical Theory of Saving". Economic Journal 38: $543-559$.

Rawls, J. (1971). A Theory of Justice. Cambridge, MA: Bellknap Press.

Sobolev, S.L. (1963). "Applications of Functional Analysis in Mathematical Physics". Translation of Mathematics Monographs, Vol 7, American Mathematical Society Providence R.I.

Solow, R. (1992). "An Almost Practical Step Toward Sustainability". Lecture on the Occasion of the Fourtieth Anniversary of Resources for the Future, Washington, DC, October.

Schipper, J. et al. (2008). "The Status of the World's Land and Marine Mammals: Diversity Threat and Knowledge". Science 322 (21): 1201-1205.

United Nations (2000). We the Peoples. Millenium Report 2000. New York.

Weizacker, C. C. von. (1967). "Lemmas for a Theory of Approximate Optimal Growth". Review of Economic Studies 34 (Jan.): 143-151.

Yosida, K. (1974). Functional Analysis. 4th ed. Berlin, Heidelberg, New York: Springer Verlag.

Yosida, K., and E. Hewitt (1952). "Finitely Level Independent Measures". Transactions of the American Mathematical Society 72: 46-66. 


\section{conomics}

The Open-Access, Open-Assessment E-Journal

\section{Please note:}

You are most sincerely encouraged to participate in the open assessment of this article. You can do so by either rating the article on a scale from 1 (bad) to 5 (excellent) or by posting your comments.

Please go to:

www.economics-ejournal.org/economics/journalarticles/2009-32

The Editor 